\title{
Motivationen, Schwierigkeiten und Chancen der Nicht-Regierungs-Organisationen bei der Bildung von Netzwerkell
}

\section{Einleitung}

Die UNCED-Konferenz 1992 in Rio de Janeiro machte den Bedeutungszuwachs der Nicht-Regierungs-Organisationen (NROn) als Akteure einer neugestalteten und verknüpften Umwelt- und Entwicklungspolitik deutlich. Die Schnittfelder und Überkreuzungen der beiden Politikbereiche zeichneten sich verstärkt seit Beginn der 90er Jahre ab, als die globalen Dimensionen der ökologischen Krise nicht mehr zu übersehen waren (Wöhlcke 1991, Harborth 1991, Ullrich/Kürzinger-Wiemann 1991, Messner 1993). Weder kann die Umweltpolitik die Nord-Süd-Problematik ausklammern noch kann die Entwicklungspolitik auf die notwendigen ökologischen Anpassungsleistungen in den Industrieländern verzichten.

Die Globalisierung der ökologischen Problemkonstellationen und die Interdependenz von Umwelt- und Entwicklungspolitik machen für die NROn veränderte Organisationsstrukturen und Handlungskonzepte erforderlich, die sich nicht zwangsläufig und eigendynamisch ergeben werden. Für den zukünftigen Einfluß der NROn wird es entscheidend darauf ankommen, wie sich ihre nationale und internationale Zusammenarbeit zukünftig gestaltet, welche Formen der Kooperation und Vernetzung sich dabei herausbilden werden. Einerseits ist es plausibel, daß die NROn durch die Bildung und Erweiterung von Netzwerken (»Verbundsystemen der Problemverarbeitung «) kosteneffizient, duplikationsvermeidend und ressourcenteilend arbeiten und ihren politischen Einfluß erhöhen können. Andererseits besteht aber auch die Gefahr, daß bei einer vorschnellen Vernetzung gerade die Qualitäten verloren gehen, die die NROn besonders auszeichnen.

Ausgehend von Überlegungen zum »Innenleben « von NROn sollen deshalb die Motivlagen, Möglichkeiten, Hemmnisse und Illusionen diskutiert werden, die sich aus einer lokalen, nationalen und internationalen Zusammenarbeit der Organisationen ergeben. Drei zentrale Fragen stehen dabei im Vordergrund: erstens, was sind die wesentlichen Problemlagen bei der Netzwerkbildung bzw. welche Voraussetzungen müssen dafür erfüllt sein, zweitens, welche Rolle können Netzwerke überhaupt spielen und drittens, wie können die NROn dadurch ihre Einflußmöglichkeiten erhöhen? 


\section{Die Rahmenbedingungen nach UNCED}

Für die NROn kann die Rio-Konferenz als wichtiger Meilenstein in ihrer Entwicklungsgeschichte verbucht werden. Erstmals gelang es den Organisationen, sich in dem Vorbereitungs-, Veranstaltungs- und Nachfolgeprozeß einer UN-Konferenz »nachhaltig « Gehör zu verschaffen und von den Medien als ernstzunehmende Größe wahrgenommen zu werden. Zwar sind die NROn noch weit von direkten Möglichkeiten der Einflußnahme entfernt, doch die gute Zusammenarbeit der zahlreichen NROn im »Global Forum «, der parallel verlaufenden Alternativ-Konferenz zur UNCED, sowie die Erfahrungen der Parallelveranstaltungen zur Weltbevölkerungskonferenz in Kairo und zur Weltbanktagung in Madrid bestärkten viele Organisationen in ihrer umwelt- und entwicklungspolitischen Arbeit.

NROn stellen jedoch kein neues Phänomen dar, sondern sind seit jeher eng mit der Entwicklung des Systems staatlicher, internationaler und transnationaler Organisationen verbunden. Sie haben in der Vergangenheit bereits an vielen internationalen Verträgen mitgewirkt, ohne jedoch einen formalen Status zu besitzen. Es bildete sich ein informeller Arbeitsmodus heraus, der als »Partizipation ohne Stimmrecht « bezeichnet wurde (Anheier 1992: 1424). US-amerikanische NROn hatten bereits 1945 bei der Gründungskonferenz der Vereinten Nationen einen offiziellen Beraterstatus und traten als Beobachter auf. Ihre Forderungen nach Partizipation schlug sich im Artikel 71 der UN-Charta nieder, der die NROn zwar vom engeren politischen Bereich der UN ausschloß, ihnen aber im Bereich des Wirtschaftsund Sozialrates eine konsultative Rolle zugestand. Anheier (1992: 1424) bewertet diese Rolle als »symptomatisch für (die) Beteiligung von NROn in anderen internationalen und supranationalen Organisationen«. Diese Einschätzung hat sich aufgrund der unzulänglichen staatlichen Bemühungen, die NROn stärker in den Konferenzprozeß einzubeziehen, auf dem Rio-Gipfel erneut als richtig erwiesen. Trotzdem kann es als eine neue Qualität der NROn bewertet werden, daß sie verstärkt als eigenständige Akteursgruppe mit eigenen Handlungsvorschlägen aufgetreten ist.

Dem Erstarken der global agierenden NRO-Community trägt die $\gg$ Agenda 21 « Rechnung, das in Rio verabschiedete »Aktionsprogramm für das 21. Jahrhundert $\ll$. Darin wird gefordert, daß »die Gesellschaft, die Regierungen und internationale Gremien Mechanismen entwickeln (sollen), die den nichtstaatlichen Organisationen die Möglichkeit geben, ihrer partnerschaftlichen Rolle im Rahmen eines umweltverträglichen und nachhaltigen Entwicklungsprozesses in verantwortlicher und wirksamer Weise gerecht zu werden « (BMU 1992: 228). Abgesehen davon, daß in dem völkerrechtlich unverbindlichen Vertragswerk keine konkreten Mechanismen zur Un- 
terstützung der NROn genannt werden, kann diese Formulierung auch so interpretiert werden, daß es um die effektivere Nutzbarmachung der NROFähigkeiten für die staatliche und suprastaatliche Umwelt- und Entwicklungspolitik geht.

Für das Erstarken der »NRO-Community « sind noch weitere Faktoren von Bedeutung. Das »verlorene Jahrzehnt« der Entwicklungspolitik, die Verschuldungskrise und die neoliberale Offensive haben zu desaströsen sozialen Schäden geführt, die zunehmend der Nachbesserung und Nachsorge bedürfen. Gleichzeitig wurden mit der sich abzeichnenden Ökologiekrise und mit dem Scheitern des langanhaltenden Modernisierungsschubes, der nach dem »zweiten Weltkrieg einsetzte und an die Grenzen der prinzipiellen Nichtverallgemeinerbarkeit des modernen westlichen Lebensstils stößt« (Simonis 1994: 480), neue Konfliktdimensionen aufgeworfen, die in den tradierten Organisationen von Politikprozessen kaum noch zu lösen sind. In diesem Sinne sind »die internationalen Netzwerke von Nicht-Regierungsorganisationen der politisch förmliche Ausdruck der Globalität der ökologischen Krise (Altvater 1994: 221) und der kapitalistischen Produktionsweise. Bei der Diskussion um eine »grassroots world order « sind diese Zusammenhänge zu berücksichtigen (vgl. dazu Bruckmeier 1994a).

\section{NROn zwischen Markt und Staat}

»NROn sind formalisierte Gebilde außerhalb von Markt und Staat, die ihre Ressourcen aus Solidaritätsbeiträgen der Gesellschaft auf der Basis von Freiwilligkeit erhalten und sie zur Bearbeitung von gesellschaftlichen Problemlagen in Kollektivgüter umformen. Soweit das NRO des Nordens tun, handelt es sich bei dem Transfer um einen länderübergreifenden und transkulturellen Vorgang « (Glagow 1992: 311).

Bei dieser Charakterisierung der NROn bleibt zwar die zunehmende Tendenz der Süd-NROn, das herrschende Konsumtions- und Produktionsverhalten in den Industrienationen der Kritik zu unterziehen, außer acht; es wird aber deutlich, daß NROn intermediär im öffentlichen, zwischenstaatlichen und transnationalen Bereich operieren, daß sie einerseits als ServiceInstitutionen für Drittparteien und Klientel fungieren und andererseits als Koalition und Verband die Interessen der Mitglieder vertreten (Anheier 1992: 1423). Damit sind die NROn ein Teil des »dritten Sektors « zwischen Markt und Staat. Allerdings ist eine genauere Verortung der Süd- und Nord-NROn in diesem Sektor notwendig, um die unterschiedlichen politischen und ökonomischen Rahmenbedingungen und damit die Ausgangssituation der NROn für eine Vernetzung angeben zu können.

Schon vor mehr als 100 Jahren wurden in den sich industrialisierenden Ländern die öffentliche Wohlfahrtseinrichtungen als ein Mittel zur Abfederung der Auswirkungen der kapitalistischen Produktionsweise 
benutzt. Soziale Spannungen, die das System hätten übermäßig strapazieren können, sollten so verhindert werden. Diese »Ventilfunktion « gewinnt in den Nationen der kapitalistischen Peripherie in dem Maße an Bedeutung, in dem sich dort die Lebensbedingungen der Menschen zunehmend verschlechtern. Ausgehend von dieser Entwicklung gewinnen die Süd-NROn erheblich an politischem Einfluß und finanziellem Gewicht, sie stellen aber keineswegs einen neuen Organisationstypus dar (Anheier 1992: 1424).

Die Süd-NROn sind vorwiegend im sozialen Bereich tätig, fördern die Selbsthilfe und zielen auf die Eigenleistungen der Bevölkerung ab. Insofern der Markt oder der Staat diese Dienste nicht erbringen kann oder will, übernehmen die NROn die Rolle einer ausgleichenden Macht zwischen diesen Sektoren, sind aber selbst wiederum abhängig von der kapitalistischen Überschußproduktion oder von staatlichen und supranationalen Subventionen und damit vom globalen politischen und ökonomischen System. Für die Entwicklungshilfe stellt Fowler daher die Forderung auf: »NGOs need to make a radical reappraisal of their development strategies and roles, as well as taking decisions on how to secure the autonomy of their funding base « (Fowler 1992: 9).

Die Zunahme an NRO-Neugründungen wird nicht selten als Folgeerscheinung des Versagens von Markt oder Staat interpretiert. Daraus wird abgeleitet, daß NROn zur Staatsentlastung und politischen Stabilisierung potentiell instabiler Gesellschaften und internationaler Systeme beitragen (Anheier 1992: 1427). Bei einer solchen Sichtweise bleiben aber andere Zusammenhänge außer acht: In den Entwicklungsländern behandeln NROn Problemlagen mit Ressourcen und Instrumenten, die weder vom Markt noch vom Staat zur Verfügung gestellt werden können (Glagow 1992: 305). Ebenso bleibt unberücksichtigt, daß auch ein politisches Kalkül damit verbunden sein kann, wenn beispielsweise der Wohnungsbau, das Erziehungswesen oder die Gesundheitsversorgung der Bevölkerung den NROn überlassen wird. Dadurch werden einerseits für Staatsaufgaben indirekt (über die NROn) Gelder aus dem Ausland akquiriert und gleichzeitig staatliche Gelder eingespart, die für andere (beispielsweise militärische) Zwecke verwendet werden können. Um dem entgegenzuwirken, müßten NROn vom Staat fordern, daß er seine Aufgaben in diesen Sektoren wahrnimmt. Aber auch dies wirft ein Problem auf: vor allem viele SüdNROn würden sich dadurch selbst überflüssig machen, weshalb die politische Einforderung staatlicher Sozialaufgaben auch nicht immer ernsthaft betrieben wird.

NROn können zudem systemgefährdend wirken, wenn zu viele Aufgaben an NROn übertragen werden oder sie beispielsweise bei der Aushandlung 
internationaler Abkommen »übermäßiges« Gewicht erhalten, womit »jenseits aller praktischen Fragen ein staatstheoretisches Problern aufgeworfen « wird (Altvater 1994: 217). Ein weiterer Aspekt, der über das Markt- und Staatsversagen hinausweist, ist darin zu sehen, daß NROn als »revolutionäre Subjekte« erscheinen können, wenn sie versuchen, den zerstörerischen Tendenzen des Kapitalismus entgegenzuwirken. Vor allem von finanzschwachen und instabilen Regierungen können NROn daher als »herrschaftsgefährdend « eingestuft werden, wenn sie nicht nur im sozialen Bereich tätig sind, sondern sich explizit als Vertreter der politischen Opposition oder einer alternativen Machtelite verstehen (Wegner 1994: 332f). Vor allem die NROn in Europa und Nordamerika haben ihre gesellschaftspolitische Integrationskraft und Stärke dieser besonderen Stellung zu verdanken. Ihr Einfluß beruht nicht auf der Großzügigkeit des Staates, sondern auf dem Widerstand, den sie gegen ihn entwickelt haben (vgl. auch Glagow 1992: 314f). Die Arbeit der umwelt- und entwicklungspolitisch ausgerichteten NROn in den Industrieländern zielt nicht mehr allein auf die materielle Versorgung marginalisierter Bevölkerungsgruppen (im Süden) ab, sondern auch auf politische Bildung und Lobbying.

Auf internationaler Ebene sind die NROn mit den verschiedenen UN-Organisationen, wie der Weltbank und dem IWF, durch verschiedene Interaktionsformen »verwoben «. Durch diese weitaus finanzkräftigsten unter den $»$ Geberorganisationen $<$, die der NRO-Community immer aufgeschlossener gegenüberstehen, entsteht die Gefahr, daß die NROn in die ausgetretenen Pfade bisheriger Entwicklungspolitik gedrängt werden. Wegner vermutet beispielsweise in der NRO-Förderpraxis der Weltbank »einen wirkungsvollen Hebel bei der Kontrolle, Marginalisierung oder völligen Ausschaltung von lokalen, staatskritischen Organisationen« (Wegner 1994: 336). Monitoring-NROn bilden mit den supranationalen Institutionen dagegen ein globales Diskussionsforum, in denen Politiken formuliert und Entscheidungen vorbereitet werden (Anheier 1992: 1425). Teilweise wurde auch eine »global community « um die Institutionen herum gebildet, um Projekte zu verändern, zu stoppen oder wenigstens öffentlich zu machen. Dadurch befinden sich die NROn in dem Dilemma, daß sie den internationalen Institutionen einerseits zuarbeiten, sich ihnen aber auch entgegenstellen und entgegen den realen Gegebenheiten den Eindruck demokratischer Verhältnisse vermitteln.

\section{Möglichkeiten einer Typologisierung}

Die NRO-Community boomt. Daran besteht mittlerweile kein Zweifel mehr. Bei allen Definitions- und Abgrenzungsproblemen von NROn ist 
festzustellen, daß sich ihre Zahl seit Ende des zweiten Weltkrieges kontinuierlich erhöht hat. Das Yearbook of International Organizations listet für Ende der 80er Jahre etwa 25000 international tätige NROn (global players) auf, deren Zahl jährlich um 2 bis 4 Prozent steigt (vgl. auch Anheier 1992). Viele der national tätigen NROn in den Peripherie-Ländern werden oft nicht registriert, so daß ihre Zahl nur geschätzt werden kann. Nuscheler (1991: 297) nennt allein für Indien 7000, die Philippinen mehrere 1000 und für Thailand mehr als 1000 Organisationen.

Um nun die Motivationen, Schwierigkeiten und Hemmnisse von NROn bei der Netzwerkbildung aufzeigen zu können, sollen zunächst die wesentlichen Merkmale der Organisationen betrachtet werden, die über die Negativ-Definition der »Nichtstaatlichkeit« hinausweisen. Dabei stehen weniger die »etablierten« NROn, wie politische Stiftungen, kirchliche Hilfswerke, Wohlfahrtsverbände und wissenschaftliche Institute im Zentrum der Überlegungen, sondern die institutionalisierten sozialen Bewegungen, die weniger strukturiert sind und kaum formelle Mitgliedschaften und formale Organisationsformen aufweisen, die aber durch eine enge Bindung an ihre Basis gekennzeichnet sind (vgl. auch Bruckmeier 1994a: 228).

Eine Betrachtung der Handlungsebenen, der Ziele und Instrumente dieser NROn, des Grades ihrer Institutionalisierung, der personellen Kapazitäten, der fachlichen Kompetenzen und der technischen sowie finanziellen Möglichkeiten können Erkenntnisse über Gemeinsamkeiten liefern, die eine Vernetzung ermöglichen bzw. über Unterschiede, die eine Vernetzung verhindern. Neben den lediglich auf nationaler Ebene agierenden NROn gibt es nach Anheier (1992: 1423) zwei weitere Organisationsformen, die international operieren: erstens die im Rahmen des Systems der UNO agierenden NROn und zweitens die NROn, die auf dem Gebiet der Entwicklungspolitik und -hilfe tätig sind. Für die vorliegenden Überlegungen greift dieser Ansatz zu kurz. Zum einen werden dadurch die international agierenden Umweltorganisationen nicht erfaßt und andererseits bleiben die international auftretenden Süd-NROn ausgeblendet. Bei Glagow (1992: 304) findet sich eine für die Untersuchung der Netzwerkbildung ebenso unzulängliche Beschreibung des Tätigkeitsspektrums durch »Kleinräumigkeit, soziale Überschaubarkeit (und) lebensweltnahe Problemlagen«. Dadurch wird zwar die Verankerung der NROn, d. h. ihre Basisnähe und deren unmittelbare (Problem-)Betroffenheit erfaßt, nicht aber, daß sie auch in die globale Systemwelt eingebettet sind.

Als übergeordnete Ziele der NROn können die Durchsetzung von Partikularinteressen, die Intervention in den historisch-politischen Prozeß oder die Errichtung neuer zivilgesellschaftlicher Strukturen, also die Herbeiführung von sozialem Wandel, benannt werden. NROn dienen dabei als Sprach- 
rohr, Vermittlungs- und Verteilungsstellen von Geld an Selbsthilfegruppen, leisten Projektarbeit, arbeiten als Menschenrechts- und Frauenverbände, sind in der Umwelt- oder Flüchtlingspolitik tätig oder wirken auf die Mobilisierung von Gegenmacht hin etc. In diesen Bereichen zeigen sich die Vorteile von NROn: Ihre Stärke liegt zweifellos in der Artikulierung der Bedürfnisse und Interessen ihrer Klientel innerhalb der lokalen, kulturellen und sozialen Gegebenheiten (Anheier 1992: 1424). Auch daß NROn meistens »partizipativ«, »sozial-integrativ«, »klientelnah«, »unbürokratisch« und »kulturell eigenständig « sind, hat zum Gründungsboom dieses Organisationstypus beigetragen. Allerdings liegen über diese $\gg$ komparativen Vorteile«, zu denen auch Freiheit von politischen Zwängen, Innovationsbereitschaft, Flexibilität sowie eine Orientierung an Arbeitsinteressen und nicht an Kapitalinteressen zählen, kaum empirische Untersuchungen vor. Das gleiche gilt für die Schwächen der NROn, zu denen die permanente Finanzknappheit, die mangelnde technische Infrastruktur oder die hohe Arbeitsbelastung der meist unbezahlten Arbeitskräfte gehören.

NROn gründen sich häufig aus normativen Motiven heraus und verfolgen vielfältige Ziele wie Bewußtseinsbildung, Einstellungsveränderung oder »capacity building «. Sie bieten zwar in manchen Fällen auch Dienstleistungen und Sachgüter an, ihre Arbeit ist aber nicht an Gewinnmaximierung, sondern am Wohl ihrer Klientel orientiert. Die Zieldefinitionen sind häufig moralisch bestimmt und folgen oft vagen, ambivalenten Zieläußerungen. Die Instrumente zur Realisierung der Ziele reichen von Mobilisierung und Sensibilisierung der Öffentlichkeit bis hin zu Lobbying sowie der Partizipation in Experten- und Entscheidungsgremien, wobei die Aktionsformen meist wichtiger sind als professionelle Expertise und Qualifizierung. Roth/Rucht weisen darauf hin, daß es

»für die neuen sozialen Bewegungen um die Entfaltung einer politischen Gegenöffentlichkeit (geht), die den Eigensinn der Protestmotive und Situationsbeschreibungen gegen die Definitionsmacht der Medien- und Verbandsöffentlichkeit zur Geltung bringt. Öffentlichkeitsformen der Bewegungen sind zugleich ihre Selbsterzeugung. Sie bilden das Medium zur Anerkennung und Verallgemeinerung von Protestmotiven, erzeugen und verbreiten 'alternative' Orientierungen, Werte und Normen und sie stellen ein Terrain dar, auf dem Opposition, Selbstbehauptung und Gegenkonzepte sich bewähren müssen« (Roth 1987: 74).

Viele Gruppen gründen sich spontan oder verlagern ihre Schwerpunkte entsprechend dem gesellschaftlich-historischen Veränderungsprozeß, was gegenüber tradierten und bürokratischen Organisationsstrukturen mit erheblichen Vorteilen verbunden ist. Während die Integration über gemeinsame Wertvorstellungen und verbindliche Programme bei fast allen NROn ähnlich ist, variiert der Grad der Institutionalisierung zwischen den verschiedenen Gruppen erheblich. Wesentliche Unterschiede zwischen NROn 
ergeben sich schließlich auch aus ihrem jeweiligen politischen, kulturellen oder religiösen Hintergund.

Hey/Brendle (1992) treffen bei den Umweltverbänden die Unterscheidung in eher »erfolgsorientierte « und eher »wertorientierte« Gruppen. Bei den »erfolgsorientierten « steht die Machbarkeit und der strategische Einsatz verschiedener Instrumente im Vordergrund, während bei den »wertorientierten « die Identität der NROn und Grundsatzfragen von größerer Bedeutung sind. Unabhängig von dieser Unterscheidung hängt das Überleben der NROn von deren Fähigkeit ab, externe finanzielle Ressourcen zu mobilisieren. Der Spendenmarkt in Verbindung mit entwicklungs- und umweltpolitischer Bildungsarbeit ist dabei eine der Haupteinnahmequellen der NROn. »Erst auf diese Weise«, so faßt Nuscheler (1991: 291) zusammen, »wurden sie zum Organisationskern einer außerparlamentarischen 'Entwicklungs-Lobby', die auf die staatliche Entwicklungspolitik Einfluß zu nehmen versucht $\ll$. Das gleiche kann auf die Umwelt-NROn bezogen werden. Andere Finanzierungsmöglichkeiten, wie etwa durch staatliche oder suprastaatliche Institutionen, erfordern dagegen oft Abstriche bei den eigenen Vorstellungen zur entwicklungs- und umweltpolitischen Arbeit, entsprechend dem afrikanischen Sprichwort: "If you have your hand in another man's pocket, you have to move when he moves«. So lassen sich NROn auch anhand ihrer externen Finanzierungsquellen und ihren Fähigkeiten zur Selbstfinanzierung typologisieren.

\section{Die Zusammenarbeit von Umwelt- und Entwicklungs-NROn}

Spätestens seit der Umweltkonferenz 1972 in Stockholm besteht ein allgemeiner Konsens, daß es eine armutsbedingte Umweltzerstörung gibt (Harborth 1991: 45). Die »ernsthafte « Verknüpfung der umwelt- und entwicklungspolitischen Fragestellung terminiert Messner (1993: 39) aber erst auf die 90er Jahre, »als die globale Dimension der Ökologiekrise offenbar wurde und als außerdem auch zunehmend Umweltprobleme in Peripheriestaaten 'entdeckt' wurden, die sich als global relevant herausstellten « (Messner 1993: 39). Mit der UNCED-Konferenz ist dann ins Bewußtsein gelangt, daß es eine reichtumsbedingte Umweltzerstörung gibt, so daß sich die verknüpften umwelt- und entwicklungspolitischen Lösungsstrategien von ihrer vorwiegend südlichen Ausrichtung lösen und damit globalisieren können. Für Altvater (1994: 191) steht Rio deshalb auch für die »beginnende Erkenntnis von der ökonomisch-ökologischen Paradoxie«.

Die Umweltbewegungen in den Industrienationen kämpften zunächst in erster Linie gegen Verschmutzungsprobleme in lokalen und nationalen Räumen. In dem Maße wie die Probleme auf der internationalen Ebene wahr- 
genommen wurden, erfolgte auch eine Berücksichtigung entwicklungspolitischer Belange. Die Forderung nach einer Ökologisierung der Entwicklungshilfe verband erstmals Umwelt-NROn und Entwicklungs-NROn. Schließlich konnte es sich auch die Umweltbewegung nicht mehr »erlauben, ihre Ziele unabhängig von der sozialen Frage (Kampf gegen Armut) und unabhängig von der politischen Frage (dem Kampf um Menschenrechte und Demokratie) zu verfolgen « (Ullrich/Kürzinger-Wiemann 1991: 168). Mit dem Begriff des »sustainable developement«, wie er im Brundtlandbericht (Hauff 1987) und dann auch im wissenschaftlichen Diskurs große Verbreitung fand, wurde schließlich ein Konzept von Entwicklung umrissen, das sowohl umweltverträglich als auch nachhaltig sein soll. Durch die Verknüpfung von Umwelt-NROn und Entwicklungs-NROn wurde ein enormer Anstieg im Mobilisierungspotential und der Einflußnahme auf die Öffentlichkeit erreicht. Ullrich/Kürzinger-Wiemann stellen dazu fest: $\gg$ Die Verletzung der Natur durch Projekte der Entwicklungszusammenarbeit bringt die öffentlichen Gemüter weit stärker auf als etwaige Unverträglichkeiten und Schäden im sozialen und ökonomischen Bereich « (Ullrich/Kürzinger-Wiemann 1991:167). Damit besteht nach Ansicht der Autoren aber auch die »Gefahr einer euro- und ökozentristischen Sichtweise «. Umweltpolitik kann in diesem Sinne dazu genutzt werden, die gescheiterte »Entwicklungshilfe « der verlorenen Jahrzehnte unter dem Vorsatz der Rettung der Natur weiterhin salonfähig zu halten, d. h. ihr eine neue Legitimationsgrundlage zu verschaffen. Damit besteht dann die Gefahr, daß die NROn des Nordens bestimmen, welche Teile der Natur unter welchen ökologischen Gesichtspunkten zu schützen sind. Andererseits gibt es aber auch die Chance, durch die Einbeziehung der Umwelt-NROn zu verbesserten entwicklungspolitischen Ansätzen zu gelangen.

\section{Das Verhältmis zwischen Süd- und Nord-NROn}

Mit der Abnahme des Übergewichts der nordamerikanischen NROn aufgrund der vermehrten Teilnahme europäischer NROn an den internationalen Aktivitäten und der Zunahme von NROn in afrikanischen, asiatischen und lateinamerikanischen Staaten wurde die Globalisierung der NROcommunity eingeleitet. Allerdings beschränkt sich diese Globalisierung noch weitgehend auf die Entwicklungszusammenarbeit (Anheier 1992: 1424). Die Süd-NROn werden "geradezu (zum) Gegenstand einer weltweit organisierten Zugriffsjagd « (Glagow 1992: 314). Sie werden verstärkt von Nord-NROn als Implementationsorgane in die Entwicklungszusammenarbeit einbezogen (Wegner 1994: 326). Diese »Maklerrolle« zwischen lokalen NROn und ausländischen Gebern verlängert die »Hilfskette vom ei- 
gentlichen Geldgeber, dem Spender oder Steuerzahler in den Industrieländern bis zu den Zielgruppen in den Entwicklungsländern« (ebd.: 341). Damit sind die potentiellen Konflikte schon angedeutet.

Die Nord-NROn treten dabei als Geberorganisationen auf, die den Verwaltungsaufwand und vor allem die Projektarbeit finanziell fördern und absichern. Sie sind damit ein $\gg$ gewichtiges Organisationselement einer internationalen und zunehmend international vernetzten NGO-Bewegung « (Nuscheler 1991: 297), die jedoch zur Umsetzung ihrer konkreten Auslandsarbeit wiederum auf die Zusammenarbeit mit den Süd-NROn angewiesen ist. Das hat für die Süd-NROn positive wie negative Folgewirkungen: die einseitige Ressourcenübertragung kann von der solidarischen Unterstützung bis zur paternalistischen Kontrollfunktion reichen. Immer aber handelt es sich um ein Abhängigkeitsverhältnis.

Bei der direkten Unterstützung durch die Nord-NROn können jedoch noch weitere Probleme auftreten. Erhalten die Süd-NROn keine zusätzlichen Gelder, sondern treten sie bei der Verteilung der Mittel in Konkurrenz zu ihren Regierungen, so ist mit einer Verschlechterung der Beziehung zwischen den NROn und dem Staat zu rechnen. ${ }^{1}$ Dadurch kann dann die Erlangung oder Aufrechterhaltung ihrer Autonomie, gerade hinsichtlich eigener Entwicklungsstrategien gefährdet werden und an deren Stelle eine »Empfängermentalität« treten. Eine Überforderung der Süd-NROn kann außerdem zu einer ungesteuerten und chaotischen Distribution von Serviceleistungen führen. Schließlich besteht die Gefahr, daß die Süd-NROn den Entscheidungen der Geberorganisationen untergeordnet und am Entscheidungsprozeß über die Projektarbeit nicht beteiligt werden. Während die Süd-NROn mit diesen Problemen konfrontiert sind, können die NordNROn übergeordnete Probleme aufgreifen, Entwicklungsperspektiven erarbeiten und dadurch die Definitionsmacht über die Ziele, Strategien und Konzepte erlangen.

Eine größere Autonomie können die Süd-NROn dadurch erreichen, daß sie mit mehreren Nord-NROn oder staatlichen Organisationen kooperieren, wodurch die Abhängigkeit aber nur umverteilt und nicht aufgehoben wird. Die Spendenakquisition vor Ort mit dem Ziel der finanziellen Unabhängigkeit dürfte für die Süd-NROn auf absehbare Zeit nur schwer möglich sein, zumal durch die Zunahme der Organisationen auch die Konkurrenz auf dem Spendenmarkt wächst. Das gleiche gilt für die Konzentration der Aktivitäten auf den produktiven Sektor, um selbst Gelder zu erwirtschaf-

Auf diese Situation reagieren die Nationen der kapitalistischen Peripherie nicht selten mit der Gruindung eigener Organisationen, den sog. Quasi-Non-Governmental-Organizations (QUANGOs) oder den Governmental-Owned-NGOs (GONGOs), um selbst Spenden und NRO-Zuwendungen von Geberländern und internationalen Organisationen zu erhalten. 
ten. Bei letzterem besteht auch die Gefahr, zu sehr in Marktnähe zu geraten und den ursprünglichen Organisationscharakter zu verlieren.

Diese Einseitigkeit in den entwicklungspolitischen Nord-Süd-Beziehungen der NROn, die sich nicht nur über den Ressourcenfluß sondern auch über die Einflußnahme bei der konkreten Projektarbeit zeigt, erfährt aber auch schon erste korrigierende Impulse. Dazu trägt bei, daß es den Süd-NROn in der Entwicklungspolitik gelungen ist, ihre Abhängigkeit vom Geld zu reduzieren und vermehrt »nicht-materielle« Hilfestellung zu geben. Gleichzeitig werden in dem Maße, wie das in den kapitalistischen Industrienationen vorherrschende Konsumtions- und Produktionsmodell von den Süd-NROn kritisiert wird, auch die Nord-NROn reagieren müssen und zwar nicht nur mit verbalen Zugeständnissen, sondern durch konkrete Maßnahmen. Die »Projektionen über eigene politische Träume, die sich im eigenen Land nicht verwirklichen lassen (Nuscheler 1991: 299) haben damit ausgedient und bieten möglicherweise die Chance für eine Demokratisierung der internationalen NRO-Beziehungen.

Diesem Prozeß werden sich die entwicklungspolitischen Nord-NROn zukünftig verstärkt stellen müssen, um nicht in ihrer politischen oder projektbezogenen Arbeit an Glaubwürdigkeit zu verlieren. In diesem Zusammenhang ist es auch wichtig, daß Kompetenzen an die NROn im Süden übertragen werden und deren Eigenständigkeit gefördert wird, so daß die »nördliche« Definitionsmacht über Probleme, Lösungskonzepte und Handlungsstrategien abgebaut werden kann. Nach Freyhold (1987: 105) muß ein Rahmen geschaffen werden, in dem »soziale Bewegungen mündig werden können und Initiativen von unten die Chance bekommen, sich dem Zugriff von Staat und Kapital zu entziehen«.

Die Nord-Süd-Beziehungen von Umwelt-NROn sind noch im Entstehen begriffen und daher weniger ideologisch vorbelastet, als dies bei den entwicklungspolitisch ausgerichteten NROn der Fall ist. Am Beispiel von Mexiko weisen Ullrich/Kürzinger-Wiemann (1991: 176f) auf einige Problemlagen hin. Vielen Organisationen fehlt es an entsprechenden Strukturen sowie an finanzieller und fachlicher Konsolidierung, was zu einer instabilen innerorganisatorischen Demokratie und fehlender Partizipation führt. »Die Verhandlungsmacht gegenüber dem Staat kann nur durch die Bildung eines Netzwerkes gestärkt werden, das nicht nur den Informationsaustausch intensivieren, die Möglichkeiten der Arbeitsteilung ausschöpfen, sondern auch Diskussionen über die Rolle der Umweltbewegung führen und Ansätze zu einer pragmatischen, alternativen Wirtschaftspolitik entwickeln kann«(Ullrich/Kürzinger-Wiemann 1991: 178). 


\section{Ansätze der Netzwerkanalyse}

Bei der Untersuchung kooperierender Organisationen kann die Netzwerkanalyse ein sinnvolles Hilfsmittel darstellen. Der Begriff des Netzwerkes ist aus der Politikfeldanalyse hervorgegangen und bezeichnet das Beziehungsgeflecht der politischen Akteure, die an einem Entscheidungsprozeß unmittelbar oder in weiterem Sinne beteiligt sind. Die Netzwerkanalyse soll in diesem Kontext einen Einblick in die Entscheidungsfindung geben und Rückschlüsse auf mögliche Politikergebnisse zulassen. So läßt sich während des UNCED-Prozesses, und dort speziell bei der Aushandlung des Klimaregimes, ein »inneres Netzwerk «, in dem die Delegationen der einzelnen Länder vertreten waren, die die Entscheidungsmacht über die Vereinbarungen hatten, von einem »äußeren Netzwerk « unterscheiden, zu dem neben den internationalen Regierungsorganisationen und den Unterorganen der Vereinten Nationen auch die NROn gehörten und das vor allem Informations- und Lobbyarbeit leistete (vgl. auch Oberthür 1994: 28f). Innerhalb der Organisationssoziologie werden Netzwerke nicht als festgefügte Einheiten betrachtet, da sie ständiger Veränderung unterliegen. Netzwerke werden sozial und kulturell von ihrer Umgebung geprägt. Keil (1992) führt hier als einfachste Beziehung zwischen zwei Organisationen die »dyadische Verbindung « auf, die auf Kooperation oder Absprache zur Angebotskoordination und -verbesserung dient. Die Erweiterung auf eine Gruppe von Organisationen führt zu dem Ansatz des Action Sets (Whetten 1987), der komplexere Interaktionsbeziehungen zwischen mehreren Organisationen untersucht. Action Sets werden auch als Teile oder Subsysteme einer Organisationspopulation betrachtet. Je größer die Anzahl der Teilnehmer einer Vernetzung ist, desto schwieriger werden eindeutige Aussagen über die Interaktionsstrukturen. Durch leistungsfähige Computerprogramme ist es zwar möglich geworden, die Interaktionsformen von Netzwerken mit vielen Teilnehmern zu modellieren, doch bleiben in solchen Untersuchungen Umwelteinflüsse, historische Dimensionen und spezifische Strukturen von Netzwerken unbeachtet.

Zur tieferen Analyse der Organisationsrealität dient́ das Konzept der Organisationskultur (Ouchi/Wilkens 1985). Die Organisationsgruppen werden dabei als Gesamtheit betrachtet, die spezifische soziale Strukturen besitzen. Hierzu werden Praktiken, Riten, Mythen und Wertvorstellungen gezählt. Durch das Konzept der Organisationskultur wird die formal-quantitativ orientierte Vernetzungsforschung zwar erweitert. Um Vernetzung auf der internationalen Ebene zu untersuchen und den dabei auftretenden Problemen der interkulturellen Kommunikation gerecht zu werden, muB die Netzwerkanalyse aber noch erheblich weiterentwickelt werden. 


\section{Der NRO-Beitrag zur Regimebildung}

Aufgrund der anhaltenden Verelendung großer Bevölkerungsteile der Erde - nicht nur in den Ländern des Südens - und der globalen Ökologiekrise, steigt die Nachfrage nach neuen internationalen entwicklungs- und umweltpolitischen Konzepten. Die unzulängliche Durchsetzungsfähigkeit tradierter, internationaler Organisationen bewirkte die Suche nach neuen Organisationsformen, die für die Globalisierung der Problemlagen entsprechende Lösungen anzubieten haben. Damit fand auch die Mitte der 80er Jahre einsetzende Diskussion um die Bildung von internationalen Regimen zunehmend Beachtung, da solche Regime als Bausteine für die Errichtung einer Neuen Weltordnung angesehen werden können (Simonis 1994: 459). Bei dem politikwissenschaftlichen Regimeansatz steht die Frage im Vordergrund, unter welchen Bedingungen sich Formen der Kooperation zur Bearbeitung von Konflikten entwickeln können und wie es zur Herausbildung, Institutionalisierung und Internalisierung von Prinzipien, Normen und Regeln kommt (vgl. Krasner 1982). Darüber hinaus erscheint es sinnvoll, die »Erfolgsbedingung « zu einer Komponente der Regimedefinition zu machen. Demnach wird nur dann von einem internationalen Regime gesprochen »wenn zwischenstaatlich vereinbarte Normen- und Regelsysteme das Handeln der beteiligten Staaten wirksam beeinflussen « (Simonis 1994: 475;vgl. auch Haggard/Simmons 1987).

In diesem Kontext bewertet Simonis (1994: 459) die UNCED-Konferenz als einen wichtigen Beitrag zur Entstehung eines Weltklimaregimes und anderer globaler Umweltregime. Bei der Aushandlung und Implementation der Vereinbarungen von Rio stehen zwar noch die Regierungen der Nationalstaaten im Zentrum, gerade im Bereich der Umwelt- und Entwicklungspolitik kommt aber auch den NROn sowie deren politischen Netzwerken eine eigenständige Rolle zu. NROn übernehmen eine wichtige Aufgabe bei der politischen und bewußtseinschaffenden Rückkopplung zwischen der internationalen und der nationalen Ebene.

Damit kommt den NROn bei der Herausbildung internationaler Regime eine wichtige Funktion zu, die sehr stark von den neuen institutionellen Arrangements abhängt, $d$. $h$. von den politisch-institutionellen Voraussetzungen für einen umfassenden Systemwandel. Eine langfristige Perspektive kann - entsprechend der Regimetheorie - nur dann verfolgt werden, wenn sich grenzüberschreitende, trans- und internationale Formen der Kooperation auch unter den NROn bilden und institutionalisieren lassen. $\gg$ Institution-building ist das Zauberwort, das nun auch auf die NRO Anwendung findet « (Glagow 1992: 306). Dabei besteht allerdings die Gefahr, daß Netzwerkstrukturen mit übergeordneten Gremien, wenn sie sich einmal in- 
stitutionalisiert haben, eine eigenständige Handlungsautonomie und Handlungslogik gewinnen und damit den Bezug zur lokalen und nationalen Basis verlieren.

Ein möglicher Beitrag der NROn zur Regimebildung wird durch die große Anzahl der unterschiedlichen Akteure sowie deren unterschiedliche Themenbereiche erschwert. Eine weitere Schwierigkeit, die schon angesprochen wurde, liegt in dem Konsensprinzip, das auch für Regime die übliche Entscheidungsfindung prägt. Dies kann dazu führen, daß es eine lange Zeit dauert, bis Entscheidungen getroffen werden und diese dann oft nur den kleinsten gemeinsamen Nenner der Beteiligten repräsentieren. Andererseits schafft die Regimebildung wiederum die institutionellen Voraussetzungen für kollektive Lern- und Konsensbildungsprozesse. Allerdings dürfte noch einige Zeit vergehen, bevor die NROn soweit an politischer Stärke gewonnen haben, daß sie nachhaltig auf Entscheidungen einwirken können und damit zu einer gewichtigen Regime-Komponente geworden sind.

\section{Der steinige Prad der Vernetzung}

Der gemeinsame Feldzug der NROn gegen die erfolglosen Entwicklungsmodelle der letzten Jahrzehnte und gegen die weltweite ökologische Zerstörung der Lebensräume - ob mit dem Ziel einer eher kosmetischen Schadensbegrenzung oder mit dem Ziel einer radikalen Veränderung - gestaltet sich äußerst schwierig. Im Hinblick auf unsere eingangs gestellten Fragen nach der Rolle, den Schwierigkeiten und den Einflußmöglichkeiten der NROn durch Netzwerkbildung sollen nun wesentliche Charakteristika, Verläufe und Eigendynamiken diskutiert werden, die für bzw. gegen eine lokale, nationale und internationale Netzwerkbildung sprechen.

Die Vorteile von Netzwerken in der Arbeitsorganisation sind zunächst evident: Durch den Austausch und die Bereitstellung von Informationen, das Abstimmen von Aktivitäten, den Aufbau einer gemeinsamen Infrastruktur und die Vermeidung von Doppelstrukturen kann das Durchsetzungspotential und die Strategiefähigkeit beträchtlich erhöht werden. Dies kann zur optimalen Ressourcenausschöpfung und Effektivierung der politischen Arbeit beitragen. Umgekehrt kann auch die Interessenbündelung zur Stärkung der einzelnen Gruppen führen. Auf der inhaltlichen Ebene kann durch die Erarbeitung gemeinsamer Forderungen und Strategieentwürfe (Konflikt- oder Kooperationsstrategien), durch eine horizontale Integration (fachliche Zusammenhänge) und eine vertikale Integration (Weitergabe der Informationen von oben nach unten) sowie durch die Verbesserung des Informationsstandes Rückhalt und Stärke vermittelt werden. 
Als Vorteil einer möglichen NRO-Vernetzung kann auch deren pluralistische Bandbreite gewertet werden, die durch das Netzwerk repräsentiert wird. Das große Spektrum der Tätigkeitsfelder der NROn stellt in diesem Zusammenhang eine Stärke ihres Organisationstypus dar. Die »Bündelung « unterschiedlicher Gruppierungen zum gleichen (Teil-)Thema dürfte um so beeindruckender wirken, je heterogener die Gruppen sind. Wenn darüber hinaus nicht nur auf lokaler und nationaler Ebene, sondern auch international die »Maschen« gestrickt werden, kann das Netzwerk zum Rahmen trans- und interkulturellen Kommunikation werden (vgl. Bruckmeier 1994: 135). Netzwerke können dadurch ein Gefühl des Zusammenhalts und der (internationalen) Solidarität vermitteln, was sich wiederum auf das Engagement und die Motivation der Mitglieder, die zum großen Teil ehrenamtlich arbeiten, positiv auswirkt.

Trotz dieser Argumente, die für eine Netzwerkbildung sprechen, lassen sich die Probleme und Nachteile nicht von der Hand weisen, die der Vernetzung widersprechen oder diese verhindern. Nicht selten sind auch eigentümliche Solidarisierungen und Rivalitäten in der NRO-Szene auszumachen. Die offensichtlichen Vorteile einer lokalen, nationalen und internationalen Vernetzung wurden in der Vergangenheit oft durch Gruppenegoismen zurückgedrängt. Da ein Beitritt zum Netzwerk auch die Reduzierung von vereinspolitischen Souveränitätsrechten, Übertragungen von Kompetenzen und die Zunahme von Arbeitsaufwand impliziert, wird den Zusammenschlüssen gegengesteuert. In der Folge kann es dann zu Konkurrenz unter den NROn oder gar unter den Netzwerken beispielsweise um Informationsvorsprünge kommen, anstatt durch das schnelle Kopieren, Faxen und Weiterleiten durchgesickerter Memos gemeinsam eine angemessene Haltung gegenüber der Weltbank (adequate position) abzusprechen (Fetscher 1994). Der Versuch, NRO-Netzwerke aufzubauen, so resümiert Nuscheler (1991: 294), scheitert jedoch nicht selten an der »Eigenbrötelei« vieler NROn.

Ein eher »erfolgsorientierter « Politikstil könnte dagegen verstärkt auf ein versöhnliches Verständnis hinwirken, so daß eine Zusammenarbeit unter NROn erleichtert wird. Vor allem bei der Suche nach einem Konsens dürfte sich diese Ausrichtung bewähren. Sie birgt jedoch die Gefahr in sich, daß durch die Aushandlung eines Minimalkonsenses und dem dabei entstehenden $\gg$ Verlust an den eigenen Inhalten « der Kontakt mit der Basis bröckelt. Die informellen Zugangsmöglichkeiten zu den politischen Entscheidungsträgern spielen bei den »erfolgsorientierten « NROn eine größere Rolle. Ein wertorientiertes Politikverständnis dagegen dürfte tendenziell eher polarisierend auf die Zusammenarbeit von NROn mit den staatlichen Stellen wirken, hat aber unter Umständen den Vorzug der erleichterten 
Identitätsbildung der Basis gegenüber der Organisation. Dem Selbstverständnis dieses Typus widerspricht meist die direkte Beeinflussung des politischen Entscheidungsprozesses. In beiden Fällen kann hingegen eine allzu starke Position oder Führungsrolle der ehrenamtlichen Mitarbeiter und der »oberen « Organisationsebene in den NROn den Aufbau von Kontakten und Netzwerken erschweren.

Die »menschliche Dimension« stellt ein besonderes Merkmal bei der Netzwerkbildung dar. Persönliche Beziehungen der RepräsentantInnen der NROn prägen oft die Vernetzung. Der starke persönliche Bezug und die zentrale Rolle der Gründer können die Kollektivität und die Kommunikation zwar in vielen Fällen erleichtern. Wenn die Vernetzung einzelner Organisationen sich aber vor allem am Engagement, den Außenkontakten und Freundschaften einzelner Personen festmacht, kann eine solche Vernetzungstruktur mit den einzelnen Personen zusammenbrechen. Das immer häufigere Auftreten von »top-campaignern « in der NRO-Szene weist bereits auf diese Schwierigkeit hin.

Die zunehmende Formalisierung, Professionalisierung und Institutionalisierung der NROn erhöhen zwar die Arbeitseffektivität und die Durchsetzungsfähigkeit, sie kann aber auch zu unerwünschter Bürokratisierung führen. Wegner (1994: 340) sieht »eine der auffälligsten Bürokratisierungstendenzen ... in der beschleunigten Entstehung von Netzwerken, NRO-Konsortien und sonstigen metropolitanen Vermittlungsinstitutionen«. Allerdings diskutiert Wegner nur Netzwerke der Entwicklungszusammenarbeit, und diese wiederum nur unter den Kriterien der Bürokratisierung und der Kosten, die sie erzeugen, nicht aber unter den Chancen, die sie bieten. Er sieht auch eher eine »institutionelle Zersplitterung des NRO-Sektors«, der durch teils gegensätzliche politisch-ideologische Orientierungen, aber vor allem durch die Konkurrenz um externe Mittel und Consultancy-Aufträge bedingt ist und sich in der geringen Neigung vieler Organisationen ausdrückt, ihre Vorhaben zu koordinieren (ebd.: 330 ).

Unter dem Blickwinkel der Maximierung von Fördergeldern, der intelligenten Einbindungsstrategie der »kleinen «, finanzschwachen NROn durch die »großen«, des Profilverlustes und des Verlustes an eigener Identität sind organisatorische Zusammenschlüsse meistens problembehaftet. Vor allem die internationale Vernetzung bei gleichzeitiger Beibehaltung des Basisbezugs bewertet Bruckmeier (1994a: 236) als schwieriges Unterfangen. Gerade aber Süd-NROn, die weniger in der entwicklungspolitischen Projektarbeit, sondern mehr im Lobbying tätig sind, plädieren verstärkt dafür, die Fähigkeit der gegenseitigen Absprache und Koordination zu verbessern, um langfristig das eigene Gewicht und das zivilisatorische Interventionspotential in internationalen Prozessen zu erhöhen. 
Da es bei der Entscheidungsfindung über Strategien und Inhalte keine Sanktionsmöglichkeiten gibt, ist die Vernetzung auf das Konsensprinzip angewiesen, was langwierige Verhandlungen erforderlich macht. Wenn diese Diskussionen dazu führen, daß sich die im Netzwerk zusammengeschlossenen NROn lediglich auf einen Minimalkonsens einigen, kann dies für viele Gruppen eine immer größere Entfernung von ihren eigentlichen Zielen und vor allem von ihrer Basis bedeuten. Gleichzeitig wird dadurch die Entstehung von »NRO-Dinosauriern« mit der Folge des Verlustes an Glaubwürdigkeit, an Altruismus und sozialem Engagement (Wegner 1994: 341) wahrscheinlicher. Wenn schlieBlich diese Netzwerke mit hierarchisch aufgebauten Dachverbänden verwechselt werden, wird auch die politische Aussagekraft der NROn verringert.

Sowohl die entwicklungs- als auch die umweltpolitischen NROn bewegen sich nicht im »herrschaftsfreien Raum«, sondern sind mit den Machtstrukturen und den Systemrestriktionen konfrontiert, die sie für eine erfolgreiche Arbeit realistisch einschätzen müssen. Die »Vorstellung, internationale Umweltprobleme durch internationale Kooperation zu lösen, klingt einleuchtend, darf jedoch nicht darüber hinwegtäuschen, daß das Gros der internationalen Umweltproblematik das Ergebnis unzureichender nationaler Umweltpolitik in den einzelnen Staaten ist « (Wöhlcke 1991: 118). Dabei besteht die »Gefahr der Verschleppung zahlreicher Probleme « und »einer Anonymisierung der Verantwortung « (ebd.: 118). Entsprechend wird in der »Entwicklungsproblematik « durch die internationale Zusammenarbeit das Bild der »Einen Welt« gezeichnet, durch das die Spezifika der sich gegenseitig bedingenden »Über- und Unterentwicklung « verwischt werden. Zumindest auf der Grundlage des technischen Niveaus globaler Kommunikation wirft die Netzwerkbildung keine größeren Probleme mehr auf, sondern scheint zahlreiche Chancen zu bieten, weshalb das Worldwatch Institute (1994: 157) in seinem neuesten Bericht $\gg Z$ Zur Lage der Welt « diesem Thema einen eigenen Abschnitt widmet: »Durch Vernetzung zu einer ökologisch nachhaltigen Entwicklung«. Diese optimistische Einschätzung begründet sich u.a. auf folgender Feststellung:

»Die elektronische Post ist ein unerläßliches Hilfsmittel für Initiativen geworden, die sich für ökologische und soziale Belange einsetzen. Tausende von Aktivisten und Organisationen rund um die Welt koordinieren ihre Kampagne heute über Rechnernetze und tauschen auf diesem Wege Nachrichten aus (ebd.: 158).

Die Computer-Netzwerke leisteten beim Rio-Gipfel einen wichtigen Beitrag zur Beteiligung der Öffentlichkeit an dem Diskussionsprozeß. Die NRO-VertreterInnen vor Ort konnten die Geschäftsstellen in ihren Herkunftsländern problemlos über den Fortgang der Konferenz informieren, gemeinsam die Positionen für das Lobbying ausarbeiten und weltweit mit 
der Presse kommunizieren. Wie Meadows u.a. (1993: 271) schreiben, kann eingewendet werden, daß die Menschen in den Peripherie-Nationen viel Wichtigeres zu tun haben, als Informationen zu verbreiten:

»Aber gerade ihre Probleme können die Unterrepräsentierten ohne Nachrichten-Netzwerke nicht angemessen formulieren. Und umgekehrt kann die Welt auch von den Leistungen der Unterrepräsentierten nicht profitieren, wenn ihre Stimmen nicht zu hören sind « (ebd.).

\section{Perspektiven der Netzwerkbildung}

Aufgrund der bisherigen Überlegungen soll eine Beurteilung zukünftiger Vernetzungsperspektiven von NROn vorgenommen werden. Dabei ist zunächst vom Diskussionsstand über die Vor- und Nachteile der Vernetzung innerhalb der NRO-Szene auszugehen. So schreibt Hermle ${ }^{2}$ : »Bekanntlich haben große, starke und gut organisierte Interessen die beste Durchsetzungschance. Wer am nachdrücklichsten auf sich aufmerksam machen kann und über die direkten Zugänge zur Administration und Exekutive verfügt, gestaltet Politik entscheidend mit« (Hermle 1994: 18).

Hilliges $^{3}$ begegnet der mit dieser positiven Einschätzung verbundenen Forderung nach mehr und leistungsfähigeren Netzwerken mit Vorsicht und warnt vor der $\gg$ Irrelevanzfalle $«$ : $\gg$ Der Zusammenschluß mehrerer Netzwerke zu einem Gesamtverband folgt dem ökonomischen Dino-Trend, spart sicher Doppelreisen, Zeit in unterschiedlichen Konferenzen mit gleichen Kosten zu gleichen Themen. Zur Nord-Süd-politischen Lobby-Arbeit und ihrer gesteigerten Durchschlagskraft sagt dieser Zusammenschluß aber erst dann etwas aus, wenn sich die tragenden Mitgliedsorganisationen intern ïber die Ziele ihrer Arbeit im Verbund und in Abgrenzung zu ihren eigenen Aufgaben Klarheit verschafft haben« (Hilliges 1994: 37). Die Auffassung von Hermle, nach dessen Ansicht das »wahlbeeinflussende Gewicht « ein bedeutsames Argument für die Vernetzung darstellt (1994: 17), orientiert sich an dem »Machbaren « und kann damit als eher »erfolgsorientiert« eingeschätzt werden. Hilliges hingegen tendiert zur Bedächtigkeit und stellt die »Wertediskussion « in den Vordergrund seiner Überlegungen. Die bisherige Diskussion zeigt, daß sich die Netzwerkbildung als Gratwanderung erweist. Zum einen muß von den NROn die Chance genutzt werden, eine kraftvolle Stimme zu entwickeln, die sich auch politisches Gehör verschaffen kann. Diese Orientierung an dem Machbaren führt aber

\footnotetext{
2 Als Leiter der Abteilung Entwicklungspolitik bei Misereor und als Sprecher der $\gg$ Plattform deutscher Nichtregierungsorganisationen bei der EG« hat Reinhard Hermle den Entwurf für ein »Entwicklungspolitisches Forum« mitverfaßt.

3 Gunther Hilliges ist Leiter der Senatsstelle für Entwicklungsarbeit in Bremen und bei Germanwatch, Terre des Hommes und Towns \& Development tätig.
} 
kaum zu den notwendigen, radikalen Veränderungen in der Umwelt- und Entwicklungspolitik, weil einer solchen Veränderung ökonomische und politische Herrschaftsinteressen entgegenstehen. Zum anderen werden sich stark wertorientierte NROn mit der Netzwerkbildung schwer tun, was zwar die Authentizität der Organisationen gewährleistet, aber womöglich die politischen Einflußmöglichkeiten schmälert. Allzugroßer Optimismus ist also unangebracht. Sinnvoll ist aber, zunächst auf intraorganisatorischer Ebene den Weg für die Vernetzung der NROn zu ebnen: »Wenn sie ihr Interventionspotential vergrößern wollen, müssen sie versuchen, durch organisatorische Beständigkeit und Sachkunde der Irrelevanzfalle zu entgehen« (Nuscheler 1991: 294). Erst darauf aufbauend kann Netzwerkbildung erfolgsversprechend sein.

Vor dem Hintergrund der diskutierten vielfältigen Problemkonstellationen muB die Strategiesuche der NROn fortgesetzt werden. Eine allgemeine Lösung für die Probleme der Netzwerkbildung wird es wohl kaum geben. Je nach den spezifischen Charakteristika der beteiligten Organisationen und der zu bearbeitenden Probleme wird dann zwischen den unterschiedlichen Formen und Dimensionen von Netzwerken gewählt werden müssen: den dicht geknïpften oder losen, den partiellen oder langfristigen, den vertikalen oder horizontalen, den intensiven oder unverbindlichen.

\section{Literatur}

Altvater, E. (1994): Die Ordnung nationaler Weltbeherrschung oder: Ein Wettbewerb von Zauberlehrlingen, in: PROKLA 95, S. 186-225.

Anheier, H. K.; Seibel, W. (Hg.) (1990): The Third Sector: Comparative Studies of Nonprofit Organizations, Berlin, New York.

Anheier, H. K. (1992): Non-governmental Organisation (NGO), in: Bauer, R. (Hg.), Lexikon des Sozial- und Gesundheitswesens, Bd. 2, München, S. 1422-1427.

Anheier, H. K. Salomon, L. M. (1993): Die internationale Systematik der Nonprofit-Organisationen, in: Bauer, R. (Hg.), Intermediäre Nonprofit Organisationen in einem neuen Europa, Rheinfelden.

Bruckmeier, K. (1994): Strategien globaler Umweltpolitik. »Umwelt und Entwicklung in den Nord-Süd-Beziehungen, Münster.

Bruckmeier, K. (1994a): Nichtstaatliche Umweltorganisationen und die Diskussionen über eine neue Weltordnung, in: PROKLA 95, S. 227-241.

Bundesministerium für Umwelt, Naturschutz und Reaktorsicherheit (BMU) (1992): Konferenz der Vereinten Nationen fiur Unwelt und Entwicklung im Juni 1992 in Rio de Janeiro. Dokumente: Agenda 21, Bonn.

Dreesmann, B. (1990): Möglichkeiten und Grenzen von Nicht-Regierungs-Organisationen für Entwicklungszusammenarbeit, in: Glagow, M. (Hg.), (1990), S. 211-218.

Elliott, Ch. (1987): Some Aspects of Relations Between the North and the South Sectors, in: World Development, Vol. 15, Supplement.

Fetscher, C. (1994): Strategien am Küchentisch, in: die tageszeitung vom 14.5.94, S. 12.

Freyhold, M. (1987): Gibt es eine Grüne Entwicklungspolitik - Kann es sie geben?, in: Peripherie, NR. 25/26, 1987, S. 93-107.

Fowler, A. (1991): Building Partnerships between Northern and Southern NGOs: Issues for the 1990', in: Development in Practice, 1: 1, S. 5-18, Oxfam. 
Fowler, A. (1992): Distant Obligations: Speculations on NGO Funding and the Global Market, in: Review of African Political Economy, No. 55, S. 9-29.

Glagow, M. (Hg.) (1984): Gesellschaftssteuerung zwischen Korporatismus und Subsidiarität, Bielefeld.

Glagow, M. (Hg.) (1990): Deutsche und internationale Entwicklungspolitik, Opladen.

Glagow, M. (1992): Die NRO in der internationalen Entwicklungszusammenarbeit, in: Handbuch der Dritten Welt, Bd. 1, Bonn, S. 304-326.

Haggard, S.; Simmons, B. A. (1987): Theories of International Regimes, in: World Politics 3 , 1987, S.491-517.

Handy, Ch. (1981): Improving Effectiveness in Voluntary Organisation, London.

Hauff, V. (Hg.) (1987): Unsere gemeinsame Zukunft. Der Brundtland-Bericht der Weltkommission für Umwelt und Entwicklung, Greven.

Hein, W. (Hg.) (1991): Umweltorientierte Entwicklungspolitik, Hamburg.

Hein, W. (Hg.) (1994): Umbruch in der Weltgesellschaft - auf dem Weg zu einer neuen Weltordnung?, Hamburg.

Hermle, R. (1994): Plädoyer für eine neue NRO-Politik, in: epd-Entwicklungspolitik, 5/94, S. 17-18.

Hey, C.; Brendle, U. (1992): Umweltverbände und EG - Handlungsmöglichkeiten der Umweltverbände für die Verbesserung des Umwelthewußtseins und der Umweltpolitik in der europäischen Gemeinschaft, Forschungsprojekt des Umweltbundesamtes, EURES Institut für regionale Studien in Europa e.V., Freiburg.

Hilliges, G. (1994): Vorsicht Irrelevanzfalle. Ziele vor neuem NRO-Verbund klären, in: epdEntwicklungspolitik, 7/8/94, S. 37-38.

Keil, M. (1992): Die Vernetzung von voluntary organizations in Indien am Beispiel Andhra Pradesh, Saarbrïcken.

Krasner, St. (1982): Structural Causes and Regime Consequences: Regimes as Intervening Variables, in: International Organization 2, S. 185-205.

Meadows, D.; u. a. (1993): Die neuen Grenzen des Wachstums, Hamburg.

Messner, F. (1993): Das Konzept der nachhaltigen Entwicklung im Dilemma internationaler Regimebildung, in: Peripherie, Nr. 51/52, S. 38-57.

Nuscheler, F. (1991): Lem- und Arbeitsbuch Entwicklungspolitik, Bonn.

Oberthür, S. (1994): Politik in Treibhaus. Die Entstehung des internationalen Klimaschutzregimes, Berlin.

Ouchi, W.; Wilkens, A. L. (1985): Organizational Culture, in: Annual Revue of Sociology 11.

Roth, R. (1987): Kommunikationsstrukturen und Vernetzungen in neuen sozialen Bewegungen, in: Roth, R.; Rucht, D.: Neue soziale Bewegungen in der Bundesrepublik Deutschland, Frankfurt/M, S. 68-88.

Seibel, W. (1992): Dritter Sektor, in: Bauer, R. (Hg.), Lexikon des Sozial- und Gesundheitswesens, Bd. 1, München, S. 455-460.

Seibel, W. (1992a): Nonprofit-Organisationen, in: Bauer, R. (Hg.), Lexikon des Sozial- und Gesundheitswesens, Bd. 2, München, S. 1427-1429.

Simonis, G. (1994): Der Erdgipfel von Rio - zu den Problemen der Institutionalisierung globaler Umweltprobleme, in: Hein, W. (Hg.), (1994), S.459-487.

Strubel, M. (1989): Umweltregime in Europa, in: Kohler-Koch, B. (Hg.), Regime in den intermationalen Beziehungen, Baden-Baden, S. 247-273.

Ullrich, D.; Kürzinger-Wiemann, E. (1991): Umweltbewegung und Entwicklungspolitik, in: Hein, W. (Hg.) (1991), S. 165-182.

Wegner, R. (1994): Zur Rolle der Nichtregierungsorganisationen in der »Neuen Weltordnung «: Entstaatlichung der Sozialpolitik oder Bürokratisierung der NRO? in: Hein, W. (Hg.) (1994), S. 325-350.

Whetten, D. A. (1987): Interorganizational Relations, in: Lorsch J. W. (1987): Handbook of Organizational Behaviour, Englewood Cliff.

Wöhlcke, M. (1991): Umweltorientierte Entwicklungspolitik: Schwierigkeiten, Widersprüche, Illusionen, in: Hein, W. (Hg.) (1991), S. 109-126.

Worldwatch Institute (1994): Zur Lage der Welt 1994. Daten für das Überleben unseres Planeten, Frankfurt a. M. 